Adrian Jose Jaimes Becerra

\title{
Evolução do veneno em cnidários baseada em dados de genomas e proteomas
}

\section{Venom evolution in cnidarians based on genomes and proteomes data}

São Paulo 


\section{Adrian Jose Jaimes Becerra}

\section{Evolução do veneno em cnidários baseada em dados de genomas e proteomas}

\section{Venom evolution in cnidarians based on genomes and proteomes data}

Dissertação apresentada ao Instituto de Biociências da Universidade de São Paulo, para a obtenção de Título de Mestre em Ciências, na Área de Zoologia.

Orientador: Prof. Dr. Antonio C. Marques

São Paulo

2015 
Jaimes-Becerra, Adrian J.

Evolução do veneno em cnidários baseada em dados de genomas e proteomas.

$103+$ VI páginas

Dissertação (Mestrado) - Instituto de Biociências da Universidade de São Paulo. Departamento de Zoologia.

1. Veneno; 2. Evolução; 3. Proteoma. 4. Genoma

I. Universidade de São Paulo. Instituto de Biociências. Departamento de Zoologia.

\section{Comissão Julgadora}

$\operatorname{Prof}(a) \operatorname{Dr}(a)$

$\operatorname{Prof}(a) \operatorname{Dr}(a)$

Prof. Dr. Antonio Carlos Marques 


\section{Resumo}

A evolução do veneno, uma das misturas mais complexas da natureza, tem sustentado o sucesso da diversificação de inúmeras linhagens de animais. Serpentes deslizantes ou medusas flutuantes utilizam o veneno, um coquetel de peptídeos farmacologicamente ativos, sais e moléculas orgânicas. Esses animais surpreendentes têm provocado grande fascínio ao longo da história humana. Nesta dissertação propomos um estudo da evolução dos venenos no filo Cnidaria, englobando dados proteômicos e genômicos. Este projeto teve como objetivos: (1) caracterizar e elucidar a evolução da composição do veneno em Cnidaria por meio da comparação de listas de proteínas; (2) testar a hipótese de que a variação na família de toxinas específica de cnidários tem sido o resultado de um regime de seleção positiva; e (3) determinar a extensão em que a duplicação de genes pode ser considerada como a principal razão para a diversificação de toxinas em Cnidaria.

O capítulo "Comparative proteomics reveals common components of a powerful arsenal in the earliest animal venomous lineage, the cnidarians" propõe o estudo comparado mais completo sobre a composição do veneno de cnidários e uma hipótese sobre a montagem evolutiva do complexo arsenal bioquímico de cnidários e do veneno ancestral desse grupo basal. Vinte e oito famílias de proteínas foram identificadas. Destas, 13 famílias foram registradas pela primeira vez no proteoma de Cnidaria. Pelo menos 15 famílias de toxinas foram recrutadas no proteoma de veneno de cnidários antes da diversificação dos grupos Anthozoa e Medusozoa.

Nos capítulos "Evidence of episodic positive selection in the evolution of jellyfish toxins of the cnidarian venom" e "Gene duplications are extensive and contribute significantly to the toxic proteome of nematocysts isolated from Acropora digitifera (Cnidaria: Anthozoa: Scleractinia)", nossas análises demonstram que as famílias de toxinas nos cnidários se diversificam amplamente mediante a duplicação de genes. Além disso, em contraste com as famílias de toxinas do veneno na maioria das linhagens animais; nós identificamos um padrão diferente na família de toxinas específica de cnidários, em que há uma seleção purificadora por longos períodos seguindo longos tempos de diversificação ou vice-versa. 


\begin{abstract}
The evolution of venom, nature's most complex concoction, has underpinned the success and diversification of numerous animal lineages. Slithering serpents or buoyant jellyfishes employ venom, a cocktail of pharmacologically active peptides, salts, and organic molecules. These astonishing animals have generated a great fascination throughout human history. In this dissertation, we propose a study of the evolution of venoms in the phylum Cnidaria, encompassing proteomic and genomic data. This project aimed: (1) to characterize and elucidate the evolution of venom composition in Cnidaria by comparing protein lists; (2) to test the hypothesis that the variation in specific family of cnidarians toxins has been the result of a positive selection regime; and (3) to determine the extent to which the genes duplication may be regarded as the main reason for the diversity of toxins in Cnidaria.

The chapter "Comparative proteomics reveals common components of a powerful arsenal in the earliest animal venomous lineage, the cnidarians" presents the most comprehensive comparative study on the cnidarians venom composition and a hypothesis about the evolutionary assembly of the complex biochemical arsenal of cnidarians and of the ancestral venom of this basal group. Twenty eight protein families were identified. Of these, 13 families were described for the first time in the proteome of Cnidaria. At least 15 types of toxin families were recruited in cnidarians venom proteome before the diversification of Anthozoa and Medusozoa groups.

In the chapters "Evidence of episodic positive selection in the evolution of jellyfish toxins of the cnidarian venom" and "Gene duplications are extensive and contribute significantly to the toxic proteome of nematocysts isolated from Acropora digitifera (Cnidaria: Anthozoa: Scleractinia)", our analyses indicate that the families of toxins in cnidarians diversify broadly through gene duplication. Besides, in contrast to the families of venom toxins in most animals lineages, we identified a different pattern in the specific family of cnidarians toxins, where there is a purifying selection for periods long, followed by long periods of diversification or vice versa.
\end{abstract}




\section{CAPÍTULO 1 \\ INTRODUCÃ̃O}

\section{O VENENO NO REINO ANIMAL}

Animais venenosos têm sido tema de fascinação ao longo da história humana, evidentemente também pelo perigo inerente associado a esses organismos (Casewell et al., 2013; Arbuckle, 2015). Igualmente, animais venenosos têm sido o objeto de numerosos estudos científicos no último século, oferecendo uma visão interessante e por vezes única em diferentes áreas biológicas (Dutertre \& Lewis, 2010; King, 2011; Harrison et al., 2011; Williams et al., 2011, Sunagar et al., 2015).

O veneno é definido como uma secreção tóxica que causa lesão fisiológica, sendo transferido passiva ou ativamente de um organismo ao meio interno de outro organismo, por meio de mecanismos de liberação e lesão mecânica (Nelsen et al., 2013). Essa definição inclui animais considerados venenosos (por exemplo, escorpiões, serpentes, aranhas e cnidários), bem como animais que não têm sido tradicionalmente reconhecidos como tais (por exemplo, sanguessugas, carrapatos, morcegos hematófagos). Ao se reconhecer analogias evolutivas de recrutamento e utilização de toxinas por parte de todos esses animais, assume-se um grande número de eventos em que o veneno evoluiu de forma independente, já que ocorre em pelo menos trinta linhagens diferentes (Fry et al., 2009a; Casewell et al., 2013). O melhor reconhecimento sobre essas expressões melhora nossa compreensão dos fatores subjacentes à evolução dos venenos e suas proteínas associadas. Paralelamente, chama a atenção para o grande conjunto de toxinas ainda não estudadas, assumidas como um grande potencial para a descoberta de moléculas bioativas.

O veneno possui múltiplas funções nas diferentes linhagens do reino animal, como predação (Fry et al., 2009b; Pekar et al., 2014), defesa (Inceoglu et al., 2003; Dutertre et al., 2014; Grow et al., 2015; Nisani \& Hayes, 2015), competição intraespecífica (Whittington et al., 2009; Macrander et al., 2015) e reprodução (Leeming, 2003) tem sido atribuídas nos distintos linhagens estudados. Esta diversidade funcional e taxonômica destaca a importância do veneno como uma inovação evolutiva. Consequentemente, uma grande variedade de estruturas evoluíram para facilitar a inoculação de venenos, como, 
bicos, dentes, arpões, nematocistos, probóscides, espinhos, sprays, e aguilhões (Fry et al., 2009a; Smith \& Wheeler, 2006; Vonk et al., 2008; Beckmann \& Ozbek, 2012).

A maioria dos venenos animais é um coquetel complexo de compostos bioativos. Os venenos compreendem tipicamente uma mistura de proteínas e peptídeos (referido como toxinas), sais e componentes orgânicos, tais como aminoácidos e neurotransmissores (Fry et al., 2009a; Hargreaves et al., 2014, Jouiaei et al., 2015b). Os componentes proteicos geralmente são os mais abundantes. A composição do veneno geralmente reflete sua função, com venenos defensivos, como em peixes ou abelhas, sendo mais simples e conservados, em que a ação principal frequentemente é uma dor localizada extrema e imediato (Church \& Hodgson, 2002; Peiren et al., 2005; de Graaf et al., 2009). Em contraste, os venenos de predadores são mais complexos e variáveis em composição e efeitos fisiológicos (Fry et al., 2009a), e essa complexidade ainda aumenta o potencial de variação na composição do veneno. Essa diversidade de composições resulta em uma variação extrema na toxicidade e no modo de ação do veneno entre táxons próximos (Mackessy, 2010), populações de uma mesma espécie (Calvete et al., 2010), sexos diferentes (Menezes et al., 2010), variações ontogenéticas na vida de um indivíduo (Andrade \& Abe, 1999) e vários outros níveis (Chippaux et al., 1991). Esses processos moldam, seja por fatores históricos ou ambientais, o conteúdo do veneno (Mackessy, 2009; Casewell et al., 2013).

A importância evolutiva e ecológica do veneno tem sido enfatizada nos últimos anos, bem como sua influência determinante sobre interações interespecíficas (Sunagar et al., 2015). Ainda, os sistemas de venenos fornecem modelos sem paralelo para investigar bases moleculares da adaptação, isto é, as inter-relações entre seleção natural e os processos genéticos e moleculares responsáveis por gerar a diversidade molecular e, portanto, a variação na composição das toxinas e sua ação (Wong \& Belov, 2012, Casewell et al., 2013; Starcevic \& Long, 2013).

\section{O VENENO NOS CNIDÁRIOS}

Os cnidários, tais como anêmonas-do-mar, corais, águas-vivas e hidras, são a mais antiga linhagem existente de animais venenosos. Desde sua origem no Neoproterozoico, há 600 milhões de anos, e portanto antes da Irradiação Cambriana, essa linhagem de anatomia simples presenciou com o surgimento e declínio de inúmeras novas formas de vida com estratégias mais complexas de sensoriamento ambiental, processamento de 
informações, locomoção e alimentação (Shinzato et al., 2007; Van Iten et al., 2014). Talvez uma parte significante do sucesso dos cnidários possa ser atribuída justamente à produção de veneno, mediador essencial na interação com presas e predadores morfologicamente mais complexos (Anderluh \& Macek, 2002; Saput, 2009; Badre, 2014).

As cnidas são organelas especializadas que definem o filo Cnidaria, capazes de descarregar seu conteúdo interno mediante a ativação dos cnidócitos por estímulos externos, mecânicos ou químicos. Cnidas contêm elementos estruturais e químicos elaborados, que atuam em diferentes funções. Cnidas estão distribuídas em várias partes do corpo dos cnidários, sendo classificadas em três tipos principais, viz., nematocistos, espirocistos e pticocistos (Östman, 2000; Özbek et al., 2009). Os nematocistos, especificamente, são encontrados em todos os cnidários, sendo a estrutura primária de inoculação do veneno nos organismos-alvo (Fautin, 2009).

Desde o início do século XX, vários experimentos analíticos e observações clínicas exploraram a diversidade toxicológica dos venenos de cnidários. A diversidade dos componentes do veneno varia desde compostos não proteicos (por exemplo, purinas, aminas biogênicas) até proteínas de peso molecular elevado, tais como enzimas que incluem proteínas lipolíticas e proteolíticas que catabolizam os tecidos da presa, toxinas que formam poros e podem causar morte celular via lise osmótica, e neurotoxinas que exibem atividades rápidas e específicas atuando sobre canais iônicos (Šuput, 2009; Mariottini \& Pane, 2013; Badré, 2014; Mariottini, 2014; Jouiaei et al., 2015b; Mariottini et al., 2015).

Como outros campos da Biologia, investigações sobre venenos foram revolucionadas nos últimos anos com abordagens da biologia de sistemas, i.e. genômica, transcriptômica e proteômica. Estudos de transcriptomas e proteomas têm mostrado que os nematocistos de vários cnidários contêm proteínas, algumas das quais únicas para o grupo, e outra já identificadas previamente para outros animais venenosos, mas poucas toxinas, de fato têm sido caracterizadas (Balasubramanian et al., 2012; Weston et al., 2013; Li et al., 2014; Rachamim et al., 2014; Brinkman et al., 2015; Gacesa et al., 2015; Jouiaei et al., 2015a; Macrander et al., 2015). Essa complexidade surpreendente e o enorme potencial dos venenos de cnidários faz surgir várias questões e possibilidades únicas para pesquisas. 


\section{OBJETIVOS GERAIS DO ESTUDO}

Listas de proteínas de oito espécies correspondentes a quatro classes de cnidários, sequências de nucleótidos e aminoácidos de uma família de toxinas especifica de cnidários e o genoma do coral Acropora digitifera, foram usadas com o objetivo de: (i) caracterizar comparativamente a composição dos diferentes venenos entre as classes de cnidários; (ii) inferir a evolução da composição do veneno entre as diferentes classes; (iii) inferir a história evolutiva e as pressões de seleção que influenciaram uma família de toxinas especifica de cnidários, (iv) determinar qual a importância de processos de duplicação gênica na formação da diversidade molecular no veneno da espécie de coral Acropora digitifera.

\section{ORGANIZACÃO DA DISSERTAC̄̃̃O}

Esta tese está organizada em cinco capítulos, sendo o primeiro esta introdução, que expõe as características gerais do estudo, seus objetivos e sua organização.

O Capítulo 2, "Comparative proteomics reveals common components of a powerful arsenal in the earliest animal venomous lineage, the cnidarians", tem como objetivo caracterizar e elucidar a evolução da composição do veneno em Cnidaria por meio da comparação de listas de proteínas resultantes das análises proteômicas de Chrysaora lactea, Tamoya haplonema e Chiropsalmus quadrumanus, geradas neste estudo, e listas de proteínas de estudos publicados anteriormente para Acropora digitifera (Gacesa et al., 2015), Olindias sambaquiensis (Weston et al., 2013), Anemonia viridis, Hydra magnipapillata e Aurelia aurita (Rachamim et al., 2014). Essa análise corresponde ao estudo comparado mais completo sobre a composição do veneno de cnidários, levantando hipóteses sobre a montagem evolutiva do complexo arsenal bioquímico de cnidários e dos venenos ancestrais desse grupo basal.

O Capítulo 3, "Evidence of episodic positive selection in the evolution of jellyfish toxins of the cnidarian venom", tem como objetivo testar a hipótese de que a variação nessa família de toxinas específica de cnidários tem sido o resultado de um regime de seleção positiva. De fato, nossas análises identificaram um padrão diferente, em que há uma seleção purificadora por longos períodos seguindo longos tempos de diversificação. Esse padrão é contrastado com a biologia do grupo e a função que nematocistos possuem a história natural de cnidários. 
O Capítulo 4, "Gene duplications are extensive and contribute significantly to the toxic proteome of nematocysts isolated from Acropora digitifera (Cnidaria: Anthozoa: Scleractinia)", é um artigo que publicamos recentemente e aqui é apresentado como anexo. Este estudo determina a extensão em que a duplicação de genes pode ser considerada como a principal razão para a diversificação de toxinas em Cnidaria. Para tal, comparamos sequências de aminoácidos das toxinas previstas e derivadas do genoma traduzido de $A$. digitifera com toxinas putativas observadas em análises proteômicas de proteínas solúveis que foram obtidas de nematocistos isolados. Concluímos que a duplicação genica desempenha um papel significativo para a diversificação de toxinas nesta espécie de coral, mas não explica a totalidade da diversidade de seu arsenal.

O Capítulo 5 traz as considerações finais deste estudo, destacando os principais resultados obtidos nos capítulos anteriores, e apresenta um marco teórico de questões que surgiram a partir desses resultados e que poderão ser abordadas em trabalhos futuros sobre evolução dos venenos em cnidários.

\section{REFERÊNCIAS}

Anderluh, G. \& Maček, P. 2002. Cytolytic peptide and protein toxins from sea anemones (Anthozoa: Actiniaria). Toxicon 40: 111-124.

Andrade, D.V. \& Abe, A.S. 1999. Relationship of venom ontogeny and diet in Bothrops. Herpetologica 55:200-204.

Arbuckle, K. 2015. Evolutionary context of venom in animals. Evolution of venemous Animals and Their Toxins 1-23.

Badré, S. 2014. Bioactive toxins from stinging jellyfish. Toxicon 91: 114-125.

Balasubramanian, P.G.; Beckmann, A.; Warnken, U.; Schnölzer, M.; Schüler, A.; Bornberg-Bauer, E.; Holstein, T.W. \& Özbek, S. 2012. Proteome of Hydra nematocyst. Journal of Biological Chemistry 287: 9672-9681.

Beckmann, A. \& Özbek, S. 2012. The nematocyst: A molecular map of the cnidarian stinging organelle. International Journal of Developmental Biology 56: 577-582.

Brinkman, D.L.; Jia, X.; Potriquet, J.; Kumar, D.; Dash, D.; Kvaskoff, D. \& Mulvenna, J. 2015. Transcriptome and venom proteome of the box jellyfish Chironex fleckeri. BMC Genomics 16: 1-15.

Calvete, J.J.; Sanz, L.; Cid, P.; de la Torre, P.; Flores-Díaz, M.; Dos Santos, M.C.; Borges, A.; Bremo, A.; Angulo, Y.; Lomonte, B.; Alape-Girón, A. \& Gutiérrez, J.M. 2010. Snake venomics of the Central American rattlesnake Crotalus simus and the South American Crotalus durissus complex points to neurotoxicity as an adaptive paedomorphic trend along Crotalus dispersal in South America. Journal of proteome research 9:528-544. 
Casewell, N.R.; Wüster, W.; Vonk, F.J.; Harrison, R.A. \& Fry, B.G. 2013. Complex cocktails: The evolutionary novelty of venoms. Trends in Ecology and Evolution 28: 219-229.

Chippaux, J.P.; Williams, V. \& White. J. 1991. Snake venom variability: Methods of study, results and interpretation. Toxicon 29:1279-303.

Church, J.E. \& Hodgson, W.C. 2002. The pharmacological activity of fish venoms. Toxicon 40:1083-1093.

De Graaf, D.C.; Aerts, M.; Danneels, E. \& Devreese, B. 2009. Bee, wasp and ant venomics pave the way for a component-resolved diagnosis of sting allergy. Journal of proteomics 72:145-154.

Dutertre, S. \& Lewis, R.J. 2010. Use of venom peptides to probe ion channel structure and function. The journal of biological chemistry 285: 13315-13320.

Dutertre, S.; Jin, A.H.; Vetter, I.; Hamilton, B.; Sunagar, K.; Lavergne, V.; Dutertre, V.; Fry, B.G.; Antunes, A.; Venter, D.J.; Alewood, P.F. \& Lewis, R.J. 2014. Evolution of separate predation- and defence-evoked venoms in carnivorous cone snails. Nature Communications 5:3521.

Fautin, D.G. 2009. Structural diversity, systematics, and evolution of cnidae. Toxicon 54: 1054-1064.

Fry, B.G.; Roelants, K.; Champagne, D.E.; Scheib, H.; Tyndall, J.D.; King, G.F.; Nevalainen, T.J.; Norman, J.; Lewis, R.J.; Norton, R.S.; Renjifo, C. \& de la Vega, R.C.R. 2009a. The toxicogenomic multiverse: convergent recruitment of proteins into animal venoms. Annual Review of Genomics and Human Genetics 10: 483511.

Fry, B.G.; Wroe, S.; Teeuwisse, W.; van Osch, M.J.P.; Moreno, K.; Ingle, J.; McHenry, C.; Ferrara, T.; Clausen, P.; Scheib, H.; Winter, K.L.; Greisman, L.; Roelants, K.; van derWeerd, L.; Clemente, C.J.; Giannakis, E.; Hodgson, W.C.; Luz, S.; Martelli, P.; Krishnasamy, K.; Kochva, E.; Kwok, H.F.; Scanlon, D.; Karas, J.; Citron, D.M.; Goldstein, E.J.C.; McNaughtan, J.E. \& Norman, J.A. 2009b. A central role for venom in predation by Varanus komodoensis (Komodo dragon) and the extinct giant Varanus (Megalania) priscus. Proceedings of the National Academy of Sciences of the United States of America 106:8969-74.

Gacesa, R.; Chung, R.; Dunn, S.R.; Weston, A.; Jaimes-Becerra, A.; Marques, A.C.; Morandini, A.; Hranueli, D.; Starcevic, A.; Ward, M. \& Long, P.F. 2015. Gene duplications are extensive and contribute significantly to the toxic proteome of nematocysts isolated from Acropora digitifera (Cnidaria: Anthozoa: Scleractinia). BMC Genomics 16:774.

Grow, N.B. \& Wirdateti, N.K.A.I. 2015. Does toxic defence in Nycticebus spp. relate to ectoparasites? The lethal effects of slow loris venom on arthropods. Toxicon 95:15.

Hargreaves, A.D.; Swain, M.T.; Logan, D.W. \& Mulley, J.F. 2014. Testing the Toxicofera: comparative reptile transcriptomics casts doubt on the single, early evolution of the reptile venom system. Toxicon 92:140-156. 
Harrison, R.A.; Cook, D.A.; Renjifo, C.; Casewell, N.R.; Currier, R.B. \& Wagstaff, S.C. 2011. Research strategies to improve snakebite treatment: challenges and progress. Journal of proteomics 74:1768-1780.

Inceoglu, B.; Lango, J.; Jing, J.; Chen, L.; Doymaz, F.; Pessah, I.N. \& Hammock, B.D. 2003. One scorpion, two venoms: prevenom of Parabuthus transvaalicus acts as an alternative type of venom with distinct mechanism of action. Proceedings of the National Academy of Sciences of the United States of America 100:922-7.

Jouiaei, M.; Sunagar, K.; Gross, A.F.; Scheib, H.; Alewood, P.F.; Moran, Y. \& Fry, B.G. 2015a. Evolution of an ancient venom: recognition of a novel family of cnidarian toxins and the common evolutionary origin of sodium and potassium neurotoxins in sea anemone. Molecular Biology and Evolution 32: 1598-1610.

Jouiaei, M.; Yanagihara, A.; Madio, B.; Nevalainen, T.; Alewood, P. \& Fry, B. 2015b. Ancient venom systems: A review on Cnidaria toxins. Toxins 7: 2251-2271.

King, G.F. 2011. Venoms as a plataform for human drugs: translating toxins into therapeutics. Expert Opinion on Biological Therapy 11: 1469-1484.

Leeming, J. 2003. Scorpions of southern Africa. South Africa: Struik Publishers.

Li, R.; Yu, H.; Xue, W.; Yue, Y.; Liu, S.; Xing, R. \& Li, P. 2014. Jellyfish venomics and venom gland transcriptomics analysis of Stomolophus meleagris to reveal the toxins associated with sting. Journal of Proteomics 106: 17-29.

Mackessy, S.P. 2009. The field of reptile toxinology: snakes, lizards and their venoms. In: Mackessy, S.P. (Ed.), Handbook of venoms and toxins of reptiles. CRC Press/Taylor \& Francis Group, Boca Raton, FL 3-23.

Mackessy, S.P. 2010. Evolutionary trends in venom composition in the western rattlesnake (Crotalus viridis sensu lato). Toxicity vs. tenderizers. Toxicon 55:14631474.

Macrander, J.; Brugler, M.R. \& Daly, M. 2015. A RNA-seq approach to identify putative toxins from acrorhagi in aggressive and non-aggressive Anthopleura elegantissima polyps. BMC Genomics 16: 1-19.

Mariottini, G.L. 2014. Haemolytic venoms from marine cnidarian jellyfish - an overview. Journal of Venom Research 5: 22-32.

Mariottini, G.L.; Bonello, G.; Giacco, E. \& Pane, L. 2015. Neurotoxic and neuroactive compounds from Cnidaria: Five decades of research.... and more. Central Nervous System Agents in Medicinal Chemistry 15: 74-80.

Mariottini, G.L. \& Pane, L. 2013. Cytotoxic and cytolytic cnidarian venoms. A review on health implications and possible therapeutic applications. Toxins 6: 108-151.

Menezes, M.C.; Furtado, M.F.; Travaglia-Cardoso, S.R.; Camargo, A.C. \& Serrano, S.M. 2006. Sex-based individual variation of snake venom proteome among eighteen Bothrops jararaca siblings. Toxicon 47:304-312.

Nelsen, D.R.; Nisani, Z.; Cooper, A.M.; Fox, G.A.; Gren, E.C.K. Corbit, A.G. \& Hayes, W.K. 2014. Poisons, toxungens, and venoms: Redefining and classifying toxic 
biological secretions and the organisms that employ them. Biological Reviews 89: $450-465$.

Nisani, Z. \& Hayes, W.K. 2015. Venom-spraying behavior of the scorpion Parabuthus transvaalicus (Arachnida: Buthidae). Behavioural Processes 115:46-52.

Östman, C. 2000. A guideline to nematocyst nomenclature and classification, and some notes on the systematic value of nematocysts. Scientia Marina 64: 31-46.

Özbek, S.; Balasubramanian, P.G. \& Holstein, T.W. 2009. Cnidocyst structure and the biomechanics of discharge. Toxicon 54: 1038-1045.

Peiren, N.; Vanrobaeys, F.; de Graaf, D.C.; Devreese, B.; Van Beeumen, J. \& Jacobs F.J. 2005. The protein composition of honeybee venom reconsidered by a proteomic approach. Biochimica et Biophysica Acta (BBA) - Proteins and Proteomics 1752: $1-5$.

Pekár, S.; Śedo, O.; Líznarová, E.; Korenko, S. \& Zdráhal, Z. 2014. David and Goliath: potent venom of an ant-eating spider (Araneae) enables capture of giant prey. Naturwissenschaften 101:533-40.

Rachamim, T.; Morgenstern, D.; Aharonovich, D.; Brekhman, V.; Lotan, T. \& Sher, D. 2014. The dynamically evolving nematocyst content of an anthozoan, a scyphozoan, and a hydrozoan. Molecular Biology and Evolution 32: 740-753.

Starcevic, A. \& Long, P.F. 2013. Diversification of animal venom peptides-were jellyfish amongst the first combinatorial chemists?. ChemBioChem 14: 1407-1409.

Sunagar, K.; Morgenstern, D.; Reitzel, A.M. \& Moran, Y. (in press). 2015. Ecological venomics: How genomics, transcriptomics and proteomics can shed new light on the ecology and evolution of venom. Journal of proteomics.

Smith, W.L. \& Wheeler, W.C. 2006. Venom evolution widespread in fishes: a phylogenetic road map for the bioprospecting of piscine venoms. Journal of heredity 97:206-217.

Šuput, D. 2009. In vivo effects of cnidarian toxins and venoms. Toxicon 54: 1190-1200.

Van Iten, H.; Marques, A.C.; Leme, J.D.M.; Pacheco, M.L.F. \& Simões, M.G. 2014. Origin and early diversification of the phylum Cnidaria Verrill: Major developments in the analysis of the taxon's Proterozoic-Cambrian history. Palaeontology 57: 677690.

Vonk, F.J.; Admiraal, J.F.; Jackson, K.; Reshef, R.; de Bakker, M.A.J.; Vanderschoot, K; Van den Berge, I.; Van Atten, M.; Burgerhout, E.; Beck, A.; Mirtschin, P.J.; Kochva, E.; Witte, F.; Fry, B.G.; Woods, A.E. \& Richardson, M.K. 2008. Evolutionary origin and development of snake fangs. Nature 454:630-633.

Weston, A.J.; Chung, R.; Dunlap, W.C.; Morandini, A.C.; Marques, A.C.; Moura-daSilva, A.M.; Ward, M.; Padilla, G.; da Silva, L.F.; Andreakis, N. \& Long, P.F. 2013. Proteomic characterisation of toxins isolated from nematocysts of the South Atlantic jellyfish Olindias sambaquiensis. Toxicon 71: 11-17. 
Whittington, C.M.; Koh ,J.M.S.; Warren, W.C.; Papenfuss, A.T.; Torres, A.M.; Kuchel, P.W. \& Belov, K. 2009. Understanding and utilising mammalian venom via a platypus venom transcriptome. Journal of Proteomics 72:155-64.

Williams, D.J.; Gutiérrez, J.M.; Calvete, J.J.; Wüster, W.; Ratanabanangkoon, K.; Paiva, O.; Brown, N.I.; Casewell, N.R.; Harrison, R.A.; Rowley, P.D.; O'Shea, M.; Jensen, S.D.; Winkel, K.D. \& Warrell, D.A. 2011. Ending the drought: new strategies for improving the flow of affordable, effective antivenoms in Asia and Africa. Journal of proteomics 74:1735-1767.

Wong, E.S.W. \& Belov, K. 2012. Venom evolution through gene duplications. Gene 496:1-7. 


\section{CAPÍTULO 5}

\section{Considerações Finais}

Embora haja avanços no conhecimento, permanecemos ignorantes sobre muitas das facetas da história natural dos venenos e das interações entre esta história natural e processos e padrões de evolução do conteúdo desses arsenais químicos. A evolução molecular das distintas famílias de toxinas no veneno, e seus mecanismos subjacentes, também são pouco compreendidos (Casewell et al., 2013; Sunagar et al., 2015). Constatando-se esse desconhecimento em grupos amplamente estudados, pode-se imaginar as deficiências de conhecimento em relação a um grupo como os cnidários, em que esforços vêm recém tomando corpo. A identificação de peptídeos tóxicos específicos em cnidários está limitada a um pequeno número de toxinas e táxons (principalmente de anêmonas-do-mar), identificados por métodos analíticos tradicionais de proteínas, com uma amostragem sub-representada (Weston et al., 2013; Li et al., 2014; Rachamim et al., 2014; Brinkman et al., 2015; Gacesa et al., 2015; Jouiaei et al., 2015a, 2015b, 2015c).

Neste estudo descrevemos três novos proteomas de dois cubozoários e um cifozoário. Estes dados, somados aos 5 proteomas anteriormente descritos, permitiram comparações mais abrangentes da composição dos venenos dos diferentes grupos. Identificamos representantes de todas as superfamílias mais importantes de proteínas tóxicas, o que reflete a grande convergência das famílias de proteínas entre todos as linhagens venenosas, ao mesmo que demonstra a complexidade do veneno de Anthozoa e Medusozoa. Das 28 famílias de proteínas identificadas neste trabalho, proteínas similares a 13 famílias foram registradas pela primeira vez no proteoma de Cnidaria. Paralelamente, estes dados permitiram elaborar a mais completa hipótese sobre o desenvolvimento evolutivo do arsenal bioquímico em cnidários, em que vislumbra-se novas perspectivas sobre a origem do veneno no grupo. Há ao menos 15 famílias de toxinas recrutadas no proteoma de veneno de cnidários antes da diversificação dos grupos Anthozoa e Medusozoa, sugerindo que os ancestrais dos cnidários já possuíam intensa atividade tóxica, em um padrão similar aos venenos atuais. 
Outras conclusões importantes deste estudo estão relacionadas à evolução molecular dos proteomas, demonstrando-se que famílias de toxinas nos cnidários se diversificam amplamente mediante à duplicação de genes, um mecanismo amplamente distribuído neste tipo de proteínas (Wong \& Belov, 2012), mas não exclusivamente por esse mecanismo. A evolução do veneno de Cnidaria também contrasta com a evolução rápida e a seleção positiva das famílias de toxinas do veneno na maioria dos linhagens animais conhecidas até o momento (Kordis \& Gubensek, 2000; Fry et al., 2009; Casewell et al., 2013). De fato, a família de toxinas específica de cnidários (jellyfish toxin, JFT) mostra um padrão diferente, com episódios de evolução diversificada que afeta poucos ramos e sítios individuais desse grupo de toxinas, o qual parece ser o padrão em linhagens mais antigas de animais venenosos (Jouiaei et al., 2015b; Sunagar \& Moran, 2015).

Caracterizações genômicas, transcriptômicas e proteômicas de veneno, coletivamente conhecidas como "venômica", têm potencial para elucidar os mecanismos moleculares que operam na evolução das famílias de genes presentes no veneno, sobretudo nos elementos que controlam sua regulação e expressão. Além disso, esta maior disponibilidade de métodos permitirá que a investigação venômica no futuro próximo proporcione novas dimensões de compreensão para a evolução e ecologia dos venenos.

\section{REFERÊNCIAS}

Brinkman, D.L.; Jia, X.; Potriquet, J.; Kumar, D.; Dash, D.; Kvaskoff, D. \& Mulvenna, J. 2015. Transcriptome and venom proteome of the box jellyfish Chironex fleckeri. BMC Genomics 16: 1-15.

Casewell, N.R.; Wüster, W.; Vonk, F.J.; Harrison, R.A. \& Fry, B.G. 2013. Complex cocktails: The evolutionary novelty of venoms. Trends in Ecology and Evolution 28: 219-229.

Fry, B.G.; Roelants, K.; Champagne, D.E.; Scheib, H.; Tyndall, J.D.; King, G.F.; Nevalainen, T.J.; Norman, J.; Lewis, R.J.; Norton, R.S.; Renjifo, C. \& de la Vega, R.C.R. 2009. The toxicogenomic multiverse: convergent recruitment of proteins into animal venoms. Annual Review of Genomics and Human Genetics 10: 483-511.

Gacesa, R.; Chung, R.; Dunn, S.R.; Weston, A.; Jaimes-Becerra, A.; Marques, A.C.; Morandini, A.; Hranueli, D.; Starcevic, A.; Ward, M. \& Long, P.F. 2015. Gene duplications are extensive and contribute significantly to the toxic proteome of nematocysts isolated from Acropora digitifera (Cnidaria: Anthozoa: Scleractinia). BMC Genomics 16:774.

Jouiaei, M.; Casewell, N.; Yanagihara, A.; Nouwens, A.; Cribb, B.; Whitehead, D.; Jackson, T.; Ali, S.; Wagstaff, S.; Koludarov, I.; Alewood, P.; Hansen, J. \& Fry, B. 2015a. Firing the sting: Chemically induced discharge of cnidae reveals novel 
proteins and peptides from box jellyfish (Chironex fleckeri) venom. Toxins 7: 936950.

Jouiaei, M.; Sunagar, K.; Gross, A.F.; Scheib, H.; Alewood, P.F.; Moran, Y. \& Fry, B.G. 2015b. Evolution of an ancient venom: recognition of a novel family of cnidarian toxins and the common evolutionary origin of sodium and potassium neurotoxins in sea anemone. Molecular Biology and Evolution 32: 1598-1610.

Jouiaei, M.; Yanagihara, A.; Madio, B.; Nevalainen, T.; Alewood, P. \& Fry, B. 2015c. Ancient venom systems: A review on Cnidaria toxins. Toxins 7: 2251-2271.

Kordis, D. \& Gubensek, F. 2000. Adaptive evolution of animal toxin multigene families. Gene 261:43-52.

Li, R.; Yu, H.; Xue, W.; Yue, Y.; Liu, S.; Xing, R. \& Li, P. 2014. Jellyfish venomics and venom gland transcriptomics analysis of Stomolophus meleagris to reveal the toxins associated with sting. Journal of Proteomics 106: 17-29.

Rachamim, T.; Morgenstern, D.; Aharonovich, D.; Brekhman, V.; Lotan, T. \& Sher, D. 2014. The dynamically evolving nematocyst content of an anthozoan, a scyphozoan, and a hydrozoan. Molecular Biology and Evolution 32: 740-753.

Sunagar, K.; Morgenstern, D.; Reitzel, A.M. \& Moran, Y. (in press). 2015. Ecological venomics: How genomics, transcriptomics and proteomics can shed new light on the ecology and evolution of venom. Journal of proteomics.

Sunagar, K \& Moran, Y. 2015. The Rise and Fall of an Evolutionary Innovation: Contrasting Strategies of Venom Evolution in Ancient and Young Animals. PLoS Genetics 11(10): e1005596.

Weston, A.J.; Chung, R.; Dunlap, W.C.; Morandini, A.C.; Marques, A.C.; Moura-daSilva, A.M.; Ward, M.; Padilla, G.; da Silva, L.F.; Andreakis, N. \& Long, P.F. 2013. Proteomic characterisation of toxins isolated from nematocysts of the South Atlantic jellyfish Olindias sambaquiensis. Toxicon 71: 11-17.

Wong, E.S.W. \& Belov, K. 2012. Venom evolution through gene duplications. Gene 496: $1-7$. 